\title{
A multicentre point prevalence survey of patterns and quality of antibiotic prescribing in Indonesian hospitals
}

\author{
Ralalicia Limato (D) ${ }^{1,2}$, Erni J. Nelwan ${ }^{3,4,5,6}$, Manzilina Mudia ${ }^{1}$, Justin de Brabander ${ }^{1}$, Helio Guterres ${ }^{3,4}$, Enty Enty ${ }^{7,8}$, \\ Ifael Y. Mauleti ${ }^{9}$, Maria Mayasari ${ }^{10}$, Iman Firmansyah ${ }^{11}$, May Hizrani ${ }^{6}$ and Raph L. Hamers $\mathbb{I D}^{1,2,4 *}$
}

${ }^{1}$ Eijkman-Oxford Clinical Research Unit, Jakarta, Indonesia; ${ }^{2}$ Centre for Tropical Medicine and Global Health, Nuffield Department of Medicine, University of Oxford, Oxford, UK; ${ }^{3}$ Department of Internal Medicine, Division of Infectious Diseases, Cipto Mangunkusumo National General Hospital, Jakarta, Indonesia; ${ }^{4}$ Faculty of Medicine, University of Indonesia, Jakarta, Indonesia; ${ }^{5}$ Infectious Disease and Immunology Research Cluster, Indonesian Medical Education and Research Institute, Jakarta, Indonesia; ${ }^{6}$ Metropolitan Medical Centre Hospital, Jakarta, Indonesia; ${ }^{7}$ Royal Taruma Hospital, Jakarta, Indonesia; ${ }^{8}$ Faculty of Medicine and Health Sciences, Atma Jaya Catholic University, Jakarta, Indonesia; ${ }^{9}$ Fatmawati General Hospital, Jakarta, Indonesia; ${ }^{10}$ St. Carolus Hospital, Jakarta, Indonesia;

${ }^{11}$ Prof. Dr. Sulianti Saroso Infectious Disease Hospital, Jakarta, Indonesia

*Corresponding author. E-mail: raph.hamers@ndm.ox.ac.uk

Received 24 January 2021; accepted 10 March 2021

Background: The global emergence of antimicrobial resistance is driven by antibiotic misuse and overuse. However, systematic data in Indonesian hospitals to adequately inform policy are scarce.

Objectives: To evaluate patterns and quality indicators of antibiotic prescribing in six general hospitals in Jakarta, Indonesia.

Methods: We conducted a hospital-wide point prevalence survey (PPS) between March and August 2019, using Global-PPS and WHO-PPS protocols. The analysis focused on antibacterials (antibiotics) for systemic use.

Results: Of 1602 inpatients, 993 (62.0\%) received $\geq 1$ antimicrobial. Of 1666 antimicrobial prescriptions, 1273 $(76.4 \%)$ were antibiotics. Indications comprised community-acquired infections (42.6\%), surgical prophylaxis (22.6\%), hospital-acquired infections (18.5\%), medical prophylaxis (9.6\%), unknown (4.6\%) and other (2.1\%). The most common reasons for antibiotic prescribing were pneumonia (27.7\%), skin and soft tissue infections $(8.3 \%)$, and gastrointestinal prophylaxis (7.9\%). The most prescribed antibiotic classes were third-generation cephalosporins (44.3\%), fluoroquinolones (13.5\%), carbapenems (7.4\%), and penicillins with $\beta$-lactamase inhibitor $(6.8 \%)$. According to the WHO AWaRe classification, Watch antibiotics accounted for $67.4 \%$, followed by $28.0 \%$ Access and $2.4 \%$ Reserve. Hospital antibiotic guidelines were not available for $28.1 \%$ of prescriptions, and, where available, guideline compliance was $52.2 \%$. Reason for the antibiotic prescription, stop/review date and planned duration were poorly documented. Culture-guided prescriptions comprised $8.1 \%$ of communityacquired infections and $26.8 \%$ of hospital-acquired infections.

Conclusions: Our data indicate a high rate of empirical use of broad-spectrum antibiotics in Indonesian hospitals, coupled with poor documentation and guideline adherence. The findings suggest important areas for antimicrobial stewardship interventions.

\section{Introduction}

Drug-resistant infections have been estimated to account for 700000 deaths per year globally, cumulating to 10 million by 2050, higher than cancer (8.2 million) and diabetes (1.5 million) combined. ${ }^{1}$ The overuse and misuse of antimicrobial agents has been well recognized as one of the key drivers of emerging antimicrobial resistance (AMR), ${ }^{2,3}$ with antimicrobial consumption projected to rise further globally. ${ }^{4}$ In response to the emerging public health crisis of AMR, the WHO has launched a global action plan, including strategies for surveillance and mitigation of antimicrobial overuse. ${ }^{5}$

Indonesia, a populous (271 million) and diverse middle-income country, is potentially an AMR hotspot, due to persistently high infectious disease burdens, including respiratory infections,

(O) The Author(s) 2021. Published by Oxford University Press on behalf of the British Society for Antimicrobial Chemotherapy.

This is an Open Access article distributed under the terms of the Creative Commons Attribution License (http://creativecommons.org/licenses/ by/4.0/), which permits unrestricted reuse, distribution, and reproduction in any medium, provided the original work is properly cited. 
diarrhoeal diseases and TB among others, ${ }^{6}$ coupled with liberal antibiotic practices and fragile health systems. ${ }^{7,8}$ The Indonesian government is increasingly supporting antimicrobial stewardship (AMS), through the national action plan on AMR launched in 2014,,${ }^{9,10}$ and as part of hospital accreditation. ${ }^{11}$ National antibiotic guidelines were released in 2011, ${ }^{12}$ but have not been updated since. However, inappropriate or unnecessary antibiotic prescribing is believed to be widespread, although systematic data are lacking to adequately inform AMS policies.

In global datasets reporting point prevalence surveys (PPS) of antibiotic use in hospitals, ${ }^{13,14}$ low and middle-income countries (LMICS) remain underrepresented. ${ }^{15,16}$ The recently introduced WHO AWaRe (Access, Watch, Reserve) antibiotic classification framework, based on accessibility versus AMR potential, is a useful metric to provide an indication of the appropriateness of antibiotic consumption. ${ }^{17,18}$

We performed a hospital-wide PPS across six acute-care, general hospitals in Jakarta, the capital city of Indonesia, with the aim of evaluating patterns and quality indicators of antibiotic prescribing. We assessed community and hospital-acquired infections as well as medical and surgical prophylaxis, by hospital, ward type and diagnosis.

\section{Patients and methods}

\section{Study design and population}

We conducted a hospital-wide PPS of antimicrobial use in a purposive sample of six hospitals across Jakarta, between March and August 2019. We followed Global PPS $(2018)^{19}$ and WHO (2019) ${ }^{13}$ protocols. Briefly, a PPS is a 'snapshot' survey to collate medical record data on antimicrobial prescriptions in hospitalized patients. Eligible patients were all hospitalized patients who received $\geq 1$ active (i.e. currently ongoing) antimicrobial by 8 a.m. on the survey day or surgical prophylaxis $\leq 24 \mathrm{~h}$ prior to the survey. We excluded emergency and day-care wards, outpatient clinics and inpatients who were discharged before or admitted after 8 a.m.

\section{Ethical considerations}

The study was approved by the Research Ethics Committee of the Faculty of Medicine of the University of Indonesia (1364/UN2.F1/ETIK/2018) and the Oxford Tropical Research Ethics Committee (559-18). The requirement for individual patient consent was waived. Permission was obtained from the hospital management or research/medical committee in each participating hospital.

\section{Data collection}

We developed a paper data collection form (DCF) comprising ward, patient and treatment sections, modified from Global-PPS ${ }^{19}$ and WHO-PPS (Appendix S1, available as Supplementary data at JAC-AMR Online). ${ }^{13}$ Data collection was conducted by one or two medical doctors from the study team, joined by one to four junior hospital doctors, who received 1 day of training and a DCF completion guideline. Each ward was completely surveyed within 1 day (to minimize the effect of patient movements) and all wards of a single hospital were surveyed within 4 weeks. De-identified data were extracted from medical notes, drug chart and/or laboratory records; if crucial data were missing or unclear (e.g. unclear writing, mismatch between diagnosis and antimicrobial treatment, missing culture result), the responsible ward nurse or clinician was asked for clarifications. The completed DCFs were entered into a study database. The data coding was verified at two stages: (i) queries during DCF completion were directly resolved with a senior team member (R.L., R.L.H.); and (ii) database inconsistencies were checked against the source data in the medical records as needed.

We included systemic antimicrobials coded on the basis of the WHO Anatomical Therapeutic Chemical (ATC) classification system as follows: antibacterials (J01), antimycotics (J02), antifungals (D01BA), antimycobacterials (J04), antivirals (J05), nitroimidazole derivatives (P01AB), intestinal anti-infectives (A07A) and antimalarials (P01B). We recorded the diagnosis/ reason for the prescribed antimicrobial (what the clinician aimed at treating or preventing), according to a diagnostic code list ${ }^{19}$ (Table S1). Antimicrobial indications were classified as: (i) community-acquired infection (CAI) if symptoms were present on admission or started $<48 \mathrm{~h}$ after admission; (ii) hospital-acquired infection (HAI) if symptoms started $\geq 48 \mathrm{~h}$ after admission; (iii) medical prophylaxis; (iv) surgical prophylaxis, categorized as single-dose, 1 day or longer than 1 day; (v) other; and (vi) unknown. We recorded the following five quality indicators of prescribing: (i) documentation of diagnosis/reason for antimicrobial use, stop/review date and treatment duration in the patient records; (ii) hospital antibiotic guideline availability (i.e. based on a review of all local guidelines by the study team) and compliance with regards to drug choice; if not available, this item was recorded as 'not assessable'; (iii) parenteral administration; (iv) culture sample taken in therapeutic use; and ( $v$ ) targeted (antibiotic prescribed in response to microbiology results) or empirical treatment.

\section{Statistical analysis}

We used descriptive statistics to summarize the data, expressed as counts or percentages, by hospital, ward type, indication and diagnosis. The analysis focused on antibacterials (antibiotics) for systemic use (ATC code J01). Antibiotics were reported by drug names, chemical class (according to the fourth level WHO ATC classification) and AWaRe groups. We used RStudio Version 1.3.1093 for all analyses.

\section{Results}

\section{Hospital characteristics}

The six participating hospitals varied by care level (four secondary, two tertiary); sector (three private, three public); availability of hospital antibiotic guidelines (five yes, one no); inclusion in the national health insurance scheme (four yes, two no). All hospitals had an antibiotic stewardship team. All 238 inpatient wards surveyed included 87 medical, 31 surgical, 95 mixed medical-surgical wards and 25 ICUs, of which there were 123 adult, 51 paediatric-neonatal and 64 mixed adult-paediatric-neonatal wards (Table 1). On the survey day, a total of 2358 active hospital beds (median 230, range 134-853 per hospital) accounted for 1602 (67.9\%) admitted patients (median 149, range 51-625 per hospital), of whom 993 (62.0\%) received $\geq 1$ antimicrobial (median 91, range 33-368 per hospital). Across the hospitals, $53.5 \%$ to $78.8 \%$ of patients received $\geq 1$ antimicrobial (Table S2).

\section{Patient characteristics}

Of 993 patients, 497 (50.1\%) were women and 782 (78.8\%) were adults, and the median age was 43 years (IQR 22-58.5; range 1 day to 99 years) (Table 2). One or more comorbidities were documented in 48.9\% (486) of patients. 299 (30.1\%) had been hospitalized in the last 90 days, and 145 (14.6\%) had been transferred from another hospital. The 993 patients receiving $\geq 1$ antimicrobial accounted for a total of 1666 active antimicrobial prescriptions (median 1 per patient, range 1-12), with 60.6\% (602) receiving one antimicrobial agent, $25.6 \%$ (254) two, and $13.8 \%$ (137) three 
Table 1. Hospital characteristics

\begin{tabular}{|c|c|c|c|c|c|c|c|}
\hline & Total & Hospital 1 & Hospital 2 & Hospital 3 & Hospital 4 & Hospital 5 & Hospital 6 \\
\hline Level of health service & - & secondary & tertiary & secondary & secondary & tertiary & secondary \\
\hline Sector & - & private & public & private & public & public & private \\
\hline Teaching hospital & - & yes & yes & yes & no & yes & no \\
\hline National health insurance scheme ${ }^{a}$ & - & no & yes & yes & yes & yes & no \\
\hline Hospital antibiotic guidelines & - & no & yes & yes & yes & yes & yes \\
\hline Inpatient wards ${ }^{\mathrm{b}}$ & 238 & 19 & 74 & 30 & 14 & 79 & 22 \\
\hline medical wards & 87 & 4 & 27 & 15 & 6 & 30 & 5 \\
\hline surgical wards & 31 & 0 & 17 & 2 & 0 & 11 & 1 \\
\hline mixed medical-surgical wards ${ }^{c}$ & 95 & 12 & 19 & 10 & 7 & 32 & 15 \\
\hline ICUs & 25 & 3 & 11 & 3 & 1 & 6 & 1 \\
\hline adult & 123 & 8 & 55 & 13 & 2 & 39 & 6 \\
\hline paediatric and/or neonatal & 51 & 5 & 12 & 6 & 3 & 21 & 4 \\
\hline mixed adult-neonatal-paediatric ${ }^{d}$ & 64 & 6 & 7 & 11 & 9 & 19 & 12 \\
\hline Inpatient beds & 2358 & 159 & 767 & 300 & 145 & 853 & 134 \\
\hline Admitted patients & $1602(67.9)$ & 100 (62.9) & $562(73.3)$ & $198(66.0)$ & $66(45.5)$ & $625(73.3)$ & $51(38.1)$ \\
\hline Admitted patients on $\geq 1$ antimicrobial & $993(62.0)$ & $75(75.0)$ & $368(65.5)$ & $106(53.5)$ & $52(78.8)$ & $359(57.4)$ & $33(64.7)$ \\
\hline medical ward & $310(31.2)$ & $47(62.7)$ & $102(27.7)$ & $45(42.5)$ & $14(26.9)$ & $95(26.5)$ & $7(21.2)$ \\
\hline surgical ward & $184(18.5)$ & $0(0.0)$ & $87(23.6)$ & $24(22.6)$ & $0(0.0)$ & $73(20.3)$ & $0(0.0)$ \\
\hline mixed medical-surgical ward ${ }^{c}$ & $401(40.4)$ & $23(30.7)$ & $135(36.7)$ & $37(34.9)$ & $35(67.3)$ & $147(40.9)$ & $24(72.7)$ \\
\hline ICU & $98(9.9)$ & $5(6.7)$ & $44(12.0)$ & $0(0.0)$ & $3(5.8)$ & $44(12.3)$ & $2(6.1)$ \\
\hline adult ward & $727(73.2)$ & $31(41.3)$ & $298(81.0)$ & $92(86.8)$ & $43(82.7)$ & $230(64.0)$ & $33(100)$ \\
\hline paediatric ward & $163(16.4)$ & $13(17.4)$ & $58(15.7)$ & $8(7.5)$ & $3(5.7)$ & $81(22.6)$ & $0(0.0)$ \\
\hline mixed adult-neonatal-paediatric ward ${ }^{d}$ & $103(10.4)$ & $31(41.3)$ & $12(3.3)$ & $6(5.7)$ & $6(11.6)$ & $48(13.4)$ & $0(0.0)$ \\
\hline
\end{tabular}

Data shown reflect the hospital situation on the survey day, and are expressed as number (percentage), unless otherwise specified.

${ }^{\mathrm{a}}$ National health insurance scheme, Jaminan Kesehatan Nasional (JKN).

bIncludes all inpatient wards in the hospital. Some wards have been further subdivided for the purpose of this survey.

'Wards that can admit both medical and surgical patients.

${ }^{\mathrm{d}}$ Wards that can admit adult, paediatric and neonatal patients.

or more. Antimicrobial use was highest in ICUs (86.8\%, 132/152), followed by surgical wards $(66.0 \%, 184 / 293)$, mixed medical-surgical wards $(65.0 \%, 401 / 622)$ and medical wards $(51.4 \%, 310 /$ 569). Concomitant use of $\geq 2$ antimicrobials was more frequent in ICUs (59.1\%, 58/98) than non-ICUs (37.2\%, 333/895) (Table S2).

\section{Antimicrobial agents prescribed}

Of 1666 antimicrobial prescriptions, $76.4 \%$ (1273) were antibiotics (J01), followed by $11.4 \%$ (197) antimycobacterials, $4.3 \%$ (72) antivirals, 3.7\% (62) antimycotics, $2.6 \%$ (43) intestinal anti-infectives, $0.8 \%(13)$ antimalarials and $0.4 \%$ (6) nitroimidazole derivatives. Among 46 different antibiotic agents (J01), the five most prescribed (accounting for $56.5 \%, 720$ ) were ceftriaxone (26.8\%, 341), levofloxacin $(10.7 \%, 137)$, metronidazole $(7.1 \%, 91)$, meropenem $(6.4 \%, 82)$ and cefotaxime $(5.6 \%, 71)$ (Table S3). The top five antibiotic classes (accounting for $78.0 \%$, 993) were third-generation cephalosporins $(44.3 \%$, 565), fluoroquinolones $(13.5 \%, 172)$, carbapenems $(7.4 \%, 94)$, penicillins with $\beta$-lactamase inhibitor $(6.8 \%$, $86)$ and aminoglycosides $(6.0 \%, 76)$ (Figure 1$)$.

\section{Reasons and indications of antibiotic prescriptions}

Table S4 summarizes diagnosis/reasons for all 1666 antimicrobial prescriptions. Among all 1273 antibiotic prescriptions (J01), the most common reasons (accounting for $49.4 \%, 629$ ) were pneumonia $(27.7 \%, 353)$, skin and soft tissue infections $(8.3 \%, 106)$, gastrointestinal prophylaxis $(7.9 \%, 101)$ and gastrointestinal infections $(5.4 \%, 69)$ (Tables 3 and S5). Ceftriaxone and levofloxacin were mainly prescribed for pneumonia and gastrointestinal infections; metronidazole for skin and soft tissue infections, intraabdominal infections and gastrointestinal prophylaxis; and meropenem for pneumonia and sepsis.

The most common antibiotic indication was CAI $(42.6 \%, 542)$, followed by surgical prophylaxis (22.6\%, 288), HAI (18.5\%, 235), medical prophylaxis $(9.6 \%, 122)$, unknown $(4.6 \%, 59)$ and other $(2.1 \%, 27)$. The top five CAI were pneumonia $(42.6 \%, 231)$, skin and soft tissue infection $(14.2 \%, 77)$, gastrointestinal infection $(12.2 \%, 66)$, sepsis $(5.5 \%, 30)$, and intra-abdominal infection $(5.4 \%, 29)$ (Table S5); top five antibiotics were ceftriaxone (32.8\%, $178)$, levofloxacin $(13.5 \%, 73)$, metronidazole $(8.1 \%, 44)$, meropenem $(7.7 \%, 42)$ and ampicillin/sulbactam $(5.4 \%, 29)$ (Table S6). Hospital-acquired pneumonia (including other HAI) was the most common HAI $(70.2 \%, 165)$, followed by intervention-related infections (including catheter-related blood stream infection, ventilator-associated pneumonia, catheter-related urinary tract infection) $(19 \%, 35)$, post-operative surgical site infection (13.6\%, 32 ) and infection present on admission from another hospital $(0.85 \%, 2)$ or long-term care facility $(0.4 \%, 1)$; no Clostridioides 
Table 2. Characteristics of patients receiving $\geq 1$ antimicrobial

\begin{tabular}{|c|c|c|c|c|c|c|c|}
\hline & $\begin{array}{c}\text { Total } \\
(n=993)\end{array}$ & $\begin{array}{c}\text { Hospital } 1 \\
(n=75)\end{array}$ & $\begin{array}{r}\text { Hospital } 2 \\
(n=368)\end{array}$ & $\begin{array}{l}\text { Hospital } 3 \\
(n=106)\end{array}$ & $\begin{array}{c}\text { Hospital } 4 \\
(n=52)\end{array}$ & $\begin{array}{l}\text { Hospital } 5 \\
(n=359)\end{array}$ & $\begin{array}{c}\text { Hospital } 6 \\
(n=33)\end{array}$ \\
\hline Female & $497(50.1)$ & $41(54.7)$ & $186(50.5)$ & $61(57.5)$ & $22(42.3)$ & $175(48.7)$ & $12(36.4)$ \\
\hline \multirow[t]{2}{*}{ Age, median $(\mathrm{IQR})^{\mathrm{a}}$} & $43(22-58.5)$ & $29(20-55)$ & $47(25-60)$ & $52(33-66)$ & $39.5(28-59)$ & $37(8-53)$ & $51(28.5-65)$ \\
\hline & $45(4.5)$ & $2(2.7)$ & $17(4.6)$ & $2(1.9)$ & $1(1.9)$ & $23(6.4)$ & $0(0.0)$ \\
\hline $1-23$ months & $63(6.3)$ & $3(4.0)$ & $20(5.4)$ & $2(1.9)$ & $1(1.9)$ & $37(10.3)$ & $0(0.0)$ \\
\hline $2-17$ years & $103(10.4)$ & $12(16.0)$ & $24(6.5)$ & $6(5.7)$ & $4(7.7)$ & $57(15.9)$ & $0(0.0)$ \\
\hline 18-29 years & $131(13.2)$ & $21(28.0)$ & $47(12.8)$ & $7(6.6)$ & $8(15.4)$ & 39 (10.9) & $9(27.3)$ \\
\hline $30-39$ years & $112(11.3)$ & 9 (12.0) & $35(9.5)$ & $24(22.6)$ & $12(23.1)$ & $30(8.4)$ & $2(6.1)$ \\
\hline $40-49$ years & $145(14.6)$ & $7(9.3)$ & 55 (14.9) & $7(6.6)$ & $8(15.4)$ & $63(17.5)$ & $5(15.2)$ \\
\hline$\geq 50$ years & $394(39.7)$ & $21(28.0)$ & $170(46.2)$ & $58(54.7)$ & $18(34.6)$ & $110(30.6)$ & $17(51.5)$ \\
\hline $\begin{array}{l}\text { National health insurance } \\
\text { holder }^{b}\end{array}$ & $743(74.8)$ & $0(0.0)$ & $329(89.4)$ & $24(22.6)$ & $50(96.2)$ & $340(94.7)$ & $0(0.0)$ \\
\hline Transfer from other hospital & $145(14.6)$ & $2(2.7)$ & $70(19.0)$ & $2(1.9)$ & $10(19.2)$ & $57(15.9)$ & $4(12.1)$ \\
\hline $\begin{array}{l}\text { Hospitalization within } \\
90 \text { days }^{c}\end{array}$ & $299(30.1)$ & $12(16.0)$ & $86(23.4)$ & $20(18.9)$ & $20(38.5)$ & $151(42.1)$ & $10(30.3)$ \\
\hline Surgery in the past 90 days $^{d}$ & $368(37.1)$ & $3(4.0)$ & $162(44.0)$ & $41(38.7)$ & $8(15.4)$ & $142(39.6)$ & $12(36.4)$ \\
\hline \multicolumn{8}{|l|}{ Catheter use } \\
\hline central vascular & $132(13.3)$ & $4(5.3)$ & $35(9.5)$ & $12(11.3)$ & $1(1.9)$ & $73(20.3)$ & $7(21.2)$ \\
\hline peripheral vascular & $941(94.8)$ & $69(92.0)$ & $357(97.0)$ & $85(80.2)$ & $51(98.1)$ & 347 (96.7) & $32(97.0)$ \\
\hline urinary & $363(36.6)$ & $9(12.0)$ & $183(49.7)$ & $35(33.0)$ & $9(17.3)$ & $119(33.1)$ & $8(24.2)$ \\
\hline intubation & $65(6.5)$ & $3(4.0)$ & $21(5.7)$ & $7(6.6)$ & 1 (1.9) & $30(8.4)$ & $3(9.1)$ \\
\hline Documented comorbidity & $486(48.9)$ & $11(14.6)$ & $209(56.8)$ & $40(37.7)$ & $41(78.8)$ & $174(48.5)$ & $14(42.4)$ \\
\hline malnutrition & $335(33.7)$ & $0(0.0)$ & $165(44.8)$ & $17(16.0)$ & $28(53.8)$ & $121(33.7)$ & $4(12.1)$ \\
\hline diabetes mellitus & $161(16.2)$ & $8(10.7)$ & $68(18.5)$ & $24(22.6)$ & $14(26.9)$ & 39 (10.9) & $8(24.2)$ \\
\hline $\mathrm{TB}$ & $120(12.1)$ & $3(4.0)$ & $37(10.1)$ & $7(6.6)$ & $24(46.2)$ & $44(12.3)$ & $5(15.2)$ \\
\hline HIV & $44(4.4)$ & $3(4.0)$ & $10(2.7)$ & $2(1.9)$ & $13(25.0)$ & $16(4.5)$ & $0(0.0)$ \\
\hline HIV on ART & $27(2.7)$ & $3(4.0)$ & $4(1.1)$ & $2(1.9)$ & $8(15.4)$ & $10(2.8)$ & $0(0.0)$ \\
\hline COPD & $11(1.1)$ & $0(0.0)$ & $7(1.9)$ & $0(0.0)$ & $3(5.8)$ & $1(0.3)$ & $0(0.0)$ \\
\hline \multicolumn{8}{|l|}{ McCabe score ${ }^{e}$} \\
\hline rapidly fatal & $29(2.9)$ & $3(4.0)$ & $8(2.2)$ & $2(1.9)$ & $0(0.0)$ & $15(4.2)$ & $1(3.0)$ \\
\hline ultimately fatal & 217 (21.9) & $3(4.0)$ & $89(24.2)$ & $32(30.2)$ & $2(3.8)$ & $84(23.4)$ & 7 (21.2) \\
\hline non-fatal & $746(75.1)$ & $69(92.0)$ & $270(73.4)$ & $72(67.9)$ & $50(96.2)$ & $260(72.4)$ & $25(75.8)$ \\
\hline unknown & $1(0.1)$ & $0(0.0)$ & $1(0.1)$ & $0(0.0)$ & $0(0.0)$ & $0(0.0)$ & $0(0.0)$ \\
\hline $\begin{array}{l}\text { Prescribed antimicrobial } \\
\text { drugs }\end{array}$ & 1666 & 114 & 630 & 158 & 98 & 622 & 44 \\
\hline median (range) per patient & $1(1-12)$ & $1(1-12)$ & $1(1-9)$ & $1(1-7)$ & $1(1-6)$ & $1(1-10)$ & $1(1-4)$ \\
\hline 1 & $602(60.6)$ & $58(77.3)$ & $204(55.4)$ & $74(69.8)$ & $32(61.5)$ & $209(58.2)$ & $25(75.8)$ \\
\hline 2 & $254(25.6)$ & $11(14.7)$ & $108(29.3)$ & $22(20.8)$ & $8(15.4)$ & $99(27.6)$ & $6(18.2)$ \\
\hline$\geq 3$ & $137(13.8)$ & $6(8.0)$ & $56(15.2)$ & $10(9.4)$ & $12(23.1)$ & $51(14.2)$ & $2(6.1)$ \\
\hline
\end{tabular}

Data shown reflect the hospital situation on the survey day, and are expressed as number (percentage), unless otherwise specified.

a Median (IQR) age was 47 (28-60) years for adults, 7 (2-11) months for children $<2$ years and 8 (3-14) days for neonates.

bJaminan Kesehatan Nasional (JKN); Unknown for 4 (0.4\%) participants.

'Before the current admission, the patient had been hospitalized in the 90 days before the survey date.

${ }^{\mathrm{d}}$ The patient underwent surgery in the past 90 days before the survey date, including surgery prior to and during the current admission.

${ }^{\mathrm{e}} \mathrm{McC}$ abe score is a simple subjective method to assess underlying illness severity and classify patients according to a prognosis of rapidly fatal ( $<1$ year), ultimately fatal (1-4 years) and non-fatal ( $>5$ years). ${ }^{37}$

difficile-associated diarrhoea was documented (Table S7). The top five antibiotics for HAI were levofloxacin $(18.7 \%, 44)$, meropenem $(13.6 \%, 32)$, ceftriaxone $(9.8 \%, 23)$, amikacin $(6.4 \%, 15)$, metronidazole and ceftazidime (6\%, 14 each) (Table S6).

The top five reasons for medical prophylaxis were neonatal (20.5\%, 25), general $(19.7 \%, 24)$, gastrointestinal $(17.2 \%, 21)$, respiratory $(15.6 \%, 19)$ and unknown $(8.2 \%, 10)$ (Table S5); the top five antibiotics were ceftriaxone $(28.7 \%, 35)$, cotrimoxazole $(17.2 \%, 21)$, gentamicin $(10.7 \%, 13)$, cefotaxime $(8.2 \%, 10)$ and ampicillin $(7.4 \%, 9)$ (Table 56$)$. The top five reasons for surgical prophylaxis were gastrointestinal $(27.8 \%, 80)$, obstetrics/gynaecology $(20.1 \%, 58)$, bone and joint $(17 \%, 49)$, urinary tract $(12.8 \%$, $37)$, central nervous system and ear-nose-throat (7.3\%, 21 each); the top five antibiotics were ceftriaxone $(26.4 \%, 76)$, cefixime 


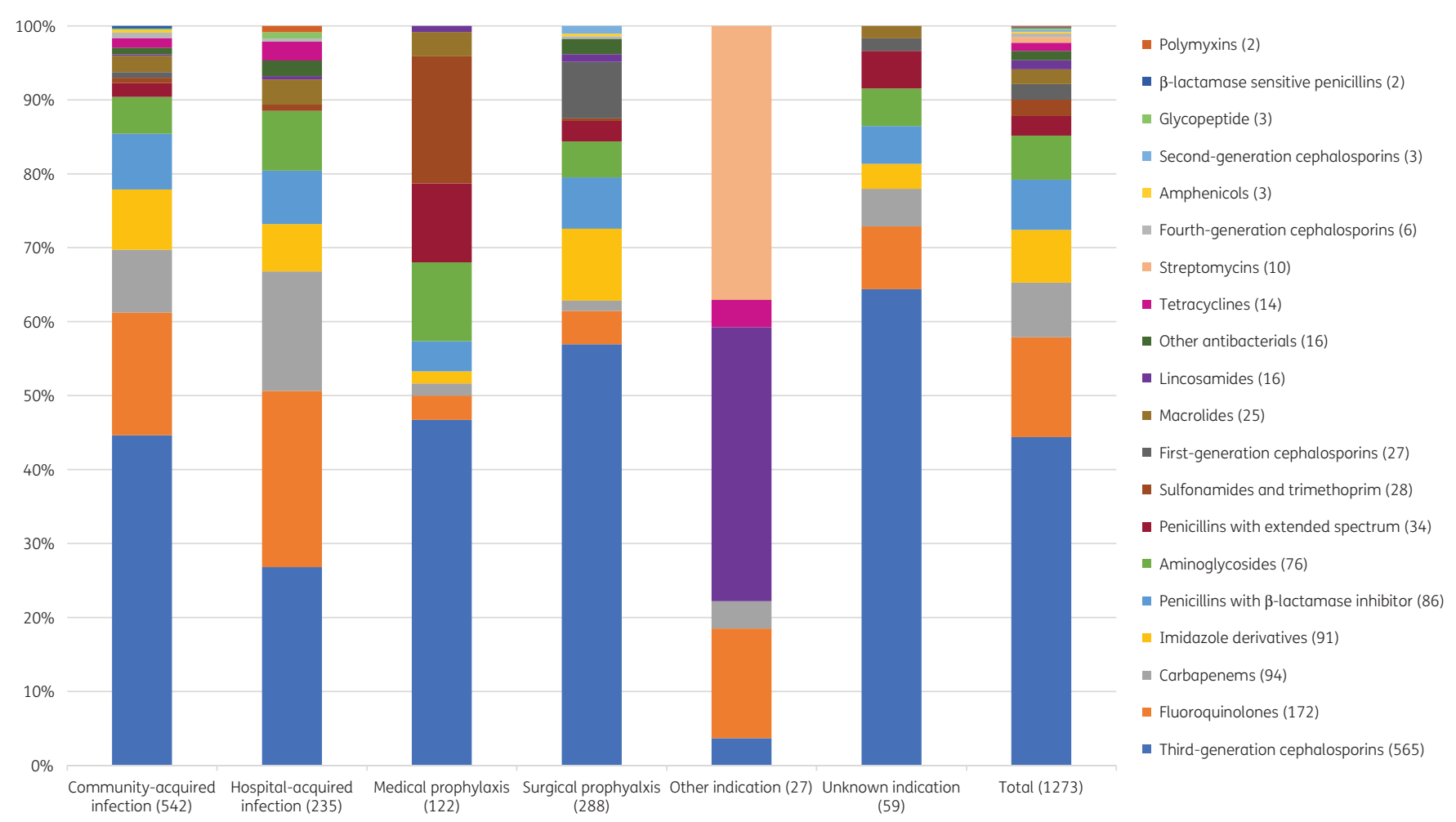

Figure 1. Systemic antibiotic use by antibiotic class, by indication.

(11.5\%, 33), cefoperazone (11.1\%, 32), metronidazole $(9.7 \%, 28)$ and cefazolin $(6.2 \%, 18)$. Notably, the duration of surgical prophylaxis was longer than 1 day for $76 \%$ (219) of prescriptions, whereas $15.0 \%$ (43) was single-dose and $9.0 \%$ (26) was for 1 day.

\section{AWaRe classification}

Of all 1273 antibiotic prescriptions (J01), 67.4\% (858) were Watch antibiotics, followed by $28.0 \%$ (356) Access, $2.4 \%$ (31) Reserve and 2.2\% (28) Unclassified (Figure 2). This pattern was similar across indications and ward types. The Watch top five were ceftriaxone (39.7\%, 341), levofloxacin $(15.8 \%, 136)$, meropenem $(9.6 \%, 82)$, cefotaxime $(8.3 \%, 71)$ and cefoperazone $(6 \%, 52)$. Notably, Watch antibiotics were commonly prescribed for the most frequent diagnoses, i.e. pneumonia $(34.4 \%, 295)$, gastrointestinal infection $(6.6 \%, 57)$ and skin and soft tissue infection $(5.8 \%, 50)$. The Access top five were metronidazole $(25.3 \%, 90)$, ampicillin/sulbactam $(12.4 \%, 44)$, gentamicin $(11.8 \%, 42)$, amikacin $(9 \%, 32)$ and amoxicillin/clavulanic acid $(8.7 \%, 31)$. Reserve antibiotics were uncommon, and included fosfomycin $(15,48.4 \%)$, tigecycline $(13,41.9 \%)$, colistin (1, 3.2\%) and linezolid (1, 3.2\%) (Figure 3, Table S8).

\section{Quality indicators of antibiotic prescribing}

\section{Documentation of antibiotic plan}

The reason for prescribing was documented for $63.5 \%$ (808/1273) of all prescriptions. Documentation of diagnosis/reason was better for therapeutic use $(546 / 777,70.3 \%)$ than prophylactic use $(230 / 410,56.0 \%)$, and in ICUs $(75.6 \%, 118 / 156)$ than non-ICUs (61.8\%, 690/1117). Stop/review date $(15.2 \%, 194 / 1273)$ and planned treatment duration $(9.8 \%, 125 / 1273)$ were poorly documented overall (Table 4).

\section{Hospital guideline availability and compliance}

Local antibiotic guidelines were not available for 28.1\% (358/1273) of prescriptions; notably, including $76.2 \%$ (93/122) of prescriptions for medical prophylaxis. Guideline compliance for drug choice was $52.2 \%$ (478/915) overall, 44.8\% (223/498) for empirical CAI treatment, $45.9 \%$ (79/172) for empirical HAI treatment, $28.5 \%$ (82/288) for surgical prophylaxis and $18.9 \%$ (23/122) for medical prophylaxis (Table 4).

\section{Parenteral use}

In total, $85.1 \%(1084 / 1273)$ of prescriptions were parenterally administered, including $88.5 \%(208 / 235)$ for HAI, $89.5 \% \%$ (486/542) for CAI, $75.4 \%$ (92/122) for medical prophylaxis and $82.3 \%$ (237/288) for surgical prophylaxis (Table 4).

\section{Culture samples taken}

Among 619 patients with $\geq 1$ antibiotic for therapeutic use, $48.8 \%$ (302) had $\geq 1$ sample taken for bacterial culture (total 831 samples, median 2, range 1-25 per patient). Blood cultures were taken in $44.4 \%(88 / 353)$ of pneumonias, $45.6 \%$ (26/57) of intra-abdominal infections, $58.6 \%(17 / 29)$ of upper urinary tract infections and $95.8 \%(23 / 24)$ of sepsis. Sputum cultures were taken in $26.9 \%$ (95/353) of pneumonias. Urine cultures were taken in $72.4 \%$ (21/29) of upper urinary tract infections (Table S9). 
Table 3. Most common diagnosis for systemic antibiotic use

\begin{tabular}{|c|c|}
\hline Diagnosis & Total $(n=1273)$ \\
\hline Pneumonia or lower respiratory tract infection & $353(27.7)$ \\
\hline Skin and soft tissue infection ${ }^{a}$ & $106(8.3)$ \\
\hline Prophylaxis for gastrointestinal infections ${ }^{b}$ & $101(7.9)$ \\
\hline Gastrointestinal infection & $69(5.4)$ \\
\hline Prophylaxis for bone and joint infection ${ }^{c}$ & $58(4.6)$ \\
\hline $\begin{array}{l}\text { Prophylaxis for obstetrics or } \\
\text { gynaecological infection }\end{array}$ & $58(4.6)$ \\
\hline Intra-abdominal infection ${ }^{d}$ & $57(4.5)$ \\
\hline Unknown reason & $47(3.7)$ \\
\hline Sepsis & $46(3.6)$ \\
\hline $\begin{array}{l}\text { Prophylaxis for urinary tract infection } \\
\text { (surgery or recurrent infection) }\end{array}$ & $42(3.3)$ \\
\hline Other diagnosis ${ }^{\mathrm{e}}$ & $35(2.7)$ \\
\hline Ear, nose, throat infection ${ }^{f}$ & $30(2.4)$ \\
\hline Upper urinary tract infection ${ }^{g}$ & $29(2.3)$ \\
\hline Central nervous system infection & $26(2.0)$ \\
\hline Medical prophylaxis for newborn risk factors & $25(2.0)$ \\
\hline
\end{tabular}

The table lists the 15 most common reasons to prescribe at least one antibiotic for systemic use (J01). Data are expressed as number (percentage) and ranked by frequency. Patients recorded with more than one diagnosis were counted by number of diagnoses. Diagnoses were coded based on the GLOBAL-PPS 2018 Diagnostic Code List (Table S1). The full list of diagnosis is shown in Table S5.

ancluding cellulitis, wound including surgical site infections, deep soft tissue not involving bone (e.g. infected pressure or diabetic ulcers, abscess).

bIncluding prophylaxis for surgery of the gastrointestinal tract, liver or biliary tree, and prophylaxis in patients with neutropenia or hepatic failure.

Including prophylaxis for surgical site infections, for plastic or orthopaedic surgery (bone or joint).

dincluding hepatobiliary, intra-abdominal abscess, etc.

${ }^{\mathrm{e}}$ Antibiotic prescribed with documentation for which there is no above diagnosis group.

fIncluding mouth, sinuses, larynx.

Including catheter-related urinary tract infection, pyelonephritis.

\section{Targeted antibiotic treatment}

Treatment was targeted in $8.1 \%(44 / 542)$ of CAI and $26.8 \%$ (63/235) of HAI; $13.0 \%$ (46/353) of pneumonias, $15.8 \%$ (9/57) of intra-abdominal infections, $44.8 \%$ (13/29) of upper urinary tract infections and $13.0 \%(6 / 46)$ of sepsis.

\section{Discussion}

This was the first contemporary hospital-wide survey in Indonesia that systematically evaluated patterns and quality of antibiotic prescribing, using the recommended PPS methodology. ${ }^{13,19}$ We demonstrated the feasibility of PPS in this low-resource setting, and generated useful data to guide local AMS interventions. We found proportions of inpatients in Indonesian hospitals receiving antibiotics to be substantially higher (62\%) than reported in global PPS datasets (27\%-39\%), which were dominated by data from high-income countries in Europe, North America and Asia. ${ }^{15,16}$ In our survey, antibiotic use varied between hospitals (53\%-79\% of patients), and was highest in ICUs (86.8\%).

Consistent with other surveys in Asia ${ }^{20,21}$ and globally, ${ }^{15}$ lower respiratory tract infections were the predominant reason for antibiotic prescribing in Jakarta hospitals. In our survey, the most-used antibiotic classes were third-generation cephalosporins (mainly ceftriaxone), fluoroquinolones (mainly levofloxacin) and carbapenems (mainly meropenem), all predominantly used for pneumonia, among several other diagnoses. Ceftriaxone was the mostused antibiotic across all major indications (i.e. CAI, HAI, surgical and medical prophylaxis). These findings are consistent with the widespread use of broad-spectrum antibiotics, predominantly third-generation cephalosporins and fluoroquinolones, in Indonesia, ${ }^{22}$ other Asian countries ${ }^{20,21,23-25}$ and globally, ${ }^{15,16}$ which may suggest that at least a proportion of these prescriptions are unnecessary or inappropriate. Moreover, empirical use of meropenem for CAI and HAI represented nearly $10 \%$ of all antibiotics for therapeutic use; this was similar to a globally reported rate of $12.2 \%,{ }^{15}$ but substantially higher than the overall $4.1 \%$ reported in European countries. ${ }^{26}$ Substantial use of carbapenems in our survey could partially be explained by the fact that two of the six hospitals were tertiary referral centres attending to complex patients, as well as high reported rates of AMR in Indonesian hospitals, particularly in common Gram-negative organisms. ${ }^{27}$ Nonetheless, culture-guided prescribing for CAI (8\%) and HAI (27\%) was low in comparison to a global study $(12 \%-27 \%$ and $20 \%-44 \%$, respectively $)^{15}$, suggesting underutilization of microbiological diagnostics as well as overuse of broad-spectrum antibiotics.

Antibiotic prescriptions for HAI (18.5\% of total), predominantly for pneumonia but also intervention-related and post-operative surgical site infections, were comparable to recent surveys in India $(19 \%)^{21}$ and Thailand (34\%), ${ }^{20}$ but considerably higher than in reports from high-income settings, e.g. ECDC survey $(6 \%)^{26}$ and the GLOBAL-PPS survey (8.4\%). ${ }^{15}$ These data confirm the significantly higher burden of HAI in LMICs compared with high-income countries.

A high proportion of antibiotic prescriptions were for surgical (23\%) and medical prophylaxis (10\%), for a range of indications. Prophylactic prescribing was unusually high for gastrointestinal infections. Prolonged ( $>1$ day) surgical prophylaxis was very common (76\%) in our survey, as has also been observed in other countries in Asia (Pakistan $97 \%,{ }^{23}$ India $77 \%,{ }^{21}$ Thailand $90 \%{ }^{20}$ ) as well as in Europe. ${ }^{15,26}$ Prolonged antibiotic prophylaxis for more than $24 \mathrm{~h}$ for most surgical indications does not prevent development of postoperative infections, compared with $<24 \mathrm{~h}$, but increases the risk of AMR and side-effects. ${ }^{28}$ Further research is warranted to explain the reasons for these patterns.

We investigated five basic quality indicators, which could be used to set benchmarks for quality improvement of antibiotic use $^{29}$ and AMS programmes. ${ }^{30}$ Documentation of the reason of prescribing (64\%) was lower than reported across studies in Europe, Asia, Africa and America (70\%-85\%). ${ }^{15,31}$ Stop or review date was poorly documented (15\%) across indications and ward types. Post-prescription review of a prescribed antimicrobial within 48-72 h of the initial order ensures appropriate choice and route of administration and optimal de-escalation (IV to oral switch) practices and prevents unnecessarily long antibiotic courses. The high (85\%) proportion of parenteral route of administration, coupled 
(a) Indication

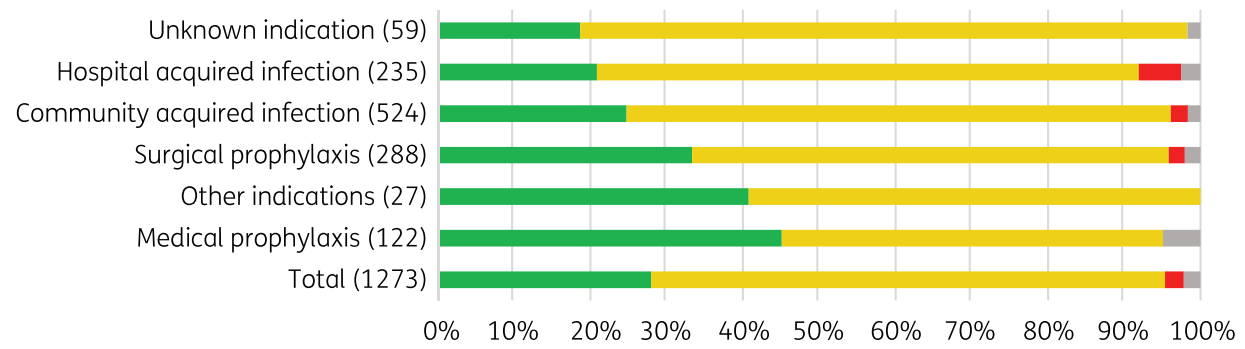

(b) Ward type

Mixed medical-surgical ward (484)

Medical ward (404)

Surgical ward (229)

Intensive Care Unit

Total (1273)

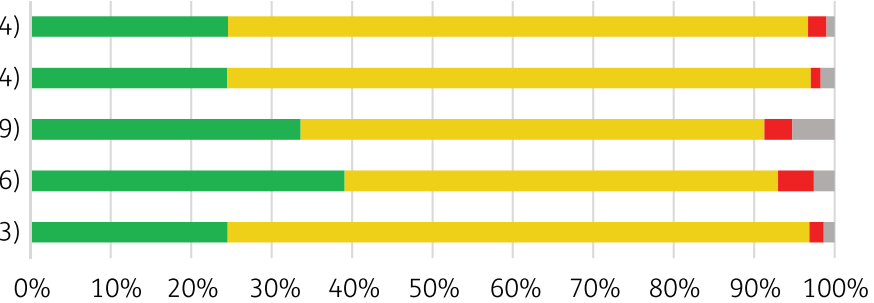

(c) Diagnosis

Genito-urinary infections (1) Eye infections (1)

PUO in non-neutropenic haemato-oncology patients (2) Pyrexia of unknown origin/PUO (3)

Pulmonary tuberculosis (14) Other diagnosis (35) Gastrointestinal infections (69)

Pneumonia or lower respiratory tract infection (353) Prophylaxis for urinary tract infections (42) Lower urinary tract infections (cystitis) (19) Febrile neutropenia (6)

Upper urinary tract infections (29) Prophylaxis for bone and joint infections (58) Unknown reason (47) Acute bronchitis or exacerbations of chronic bronchitis (4) General medical prophylaxis (24) Prophylaxis for central nervous system infections (24) Prophylaxis for ear, nose, throat infections (22) Bone and joint Infections (13) Prophylaxis for obstetrics or gynaecological infections (58) Bacteraemia (3) Upper respiratory tract viral infection (9) Central nervous system infections (26) Sepsis (46) Prophylaxis for gastrointestinal infections (101)

Ear, nose, throat infections (30) Skin and soft tissue infections (106) Intra-abdominal infections (57) Medical prophylaxis for maternal risk factors (4) Obstetrics/gynaecological infections (2) Prophylaxis for eye operations (5) Prophylaxis for cardiac or vascular infections (8) Prophylaxis for respiratory infections (20) Cardiovascular system infections (7)

Medical prophylaxis for new-born risk factors (25)
Total (1273)
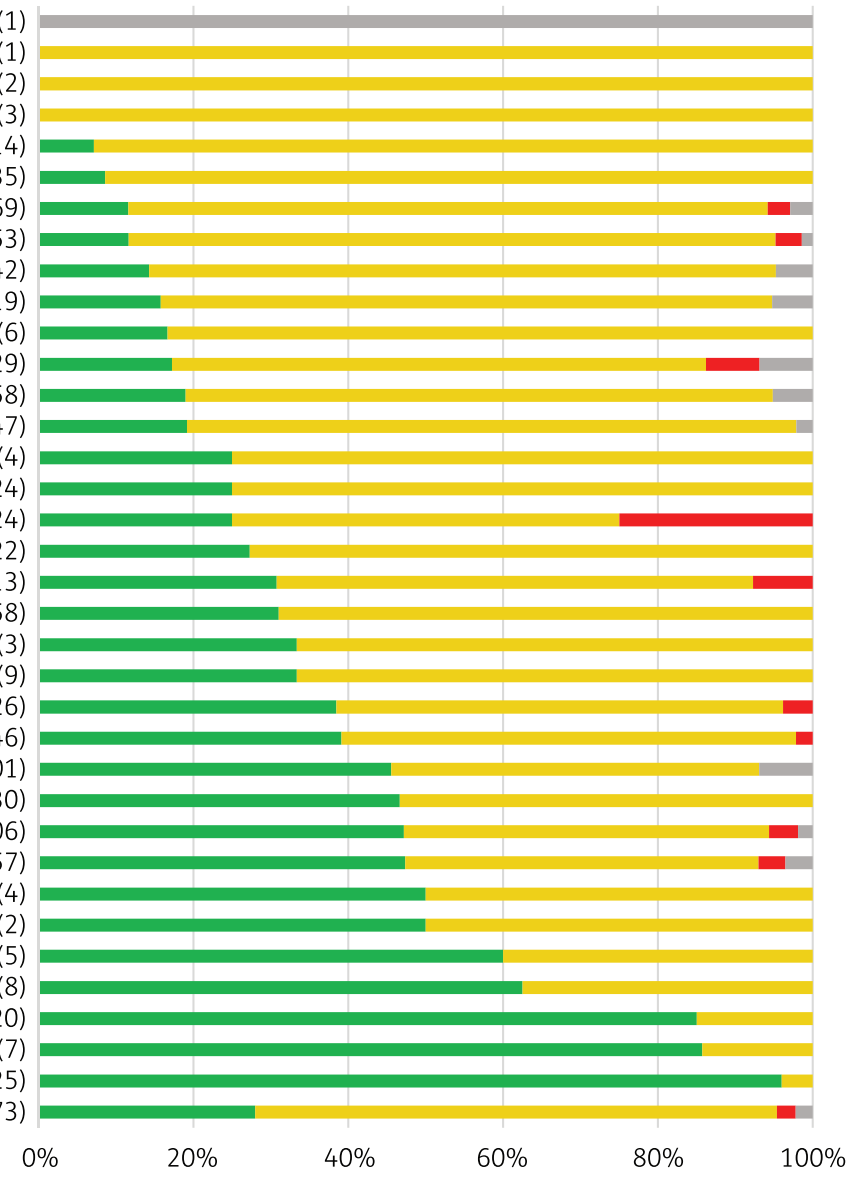

-Access Watch $\square$ Reserve Unclassified

Figure 2. Systemic antibiotic use by AWaRe classification.

with high rates of empirical therapy and suboptimal use of microbiological cultures, suggests lack of de-escalation protocols in the participating hospitals. Proactive IV to oral switching policies are recognized as a key metric for AMS processes, and can reduce catheter-related complications, healthcare costs and duration of hospital stays. ${ }^{32}$

A systematic review and meta-analysis showed that guidelineadherent empirical therapy was associated with a relative risk 


\begin{tabular}{|c|c|c|c|c|c|c|c|c|c|c|c|c|c|}
\hline \multirow[b]{2}{*}{$\begin{array}{l}\text { Total antibiotic use } \\
(n=1273) \\
90.6 \% \text { DU }\end{array}$} & & \multicolumn{4}{|c|}{ Therapeutic use } & \multicolumn{4}{|c|}{ Prophylactic use } & \multirow{2}{*}{\multicolumn{2}{|c|}{$\begin{array}{c}\text { Other indication } \\
(n=27) \\
85.1 \% \mathrm{DU} \\
\end{array}$}} & \multirow{2}{*}{\multicolumn{2}{|c|}{$\begin{array}{c}\text { Unknown indication } \\
(n=59) \\
83.2 \% \mathrm{DU}\end{array}$}} \\
\hline & & $\begin{array}{c}\text { CAI } \\
(n=542) \\
89.7 \% \text { DU }\end{array}$ & & $\begin{array}{c}\text { HAI } \\
(n=235) \\
89.4 \% \text { DU }\end{array}$ & & $\begin{array}{c}\text { Medical prophy } \\
(n=122) \\
92 \% \mathrm{DU}\end{array}$ & & $\begin{array}{r}\text { Surgical prophy } \\
(n=288) \\
91.4 \% \mathrm{DU}\end{array}$ & & & & & \\
\hline Ceftriaxone & $341(26.8)$ & Ceftriaxone & $178(32.8)$ & Levofloxacin & $44(18.7)$ & Ceftriaxone & $35(28.7)$ & Ceftriaxone & $76(26.4)$ & Clindamycin & $10(37.0)$ & Ceftriaxone & $29(49.2)$ \\
\hline Levofloxacin & $136(10.7)$ & Levofloxacin & $73(13.5)$ & Meropenem & $32(13.6)$ & Cotrimoxazole & $21(17.2)$ & Cefixime & $33(11.5)$ & Streptomycin & $10(37.0)$ & Cefotaxime & $7(11.9)$ \\
\hline Metronidazole & $90(7.1)$ & Metronidazole & $44(8.1)$ & Ceftriaxone & $23(9.8)$ & Gentamicin & $13(10.7)$ & Cefoperazone & $32(11.1)$ & Levofloxacin & $3(11.1)$ & Levofloxacin & $5(8.5)$ \\
\hline Meropenem & $82(6.4)$ & Meropenem & $42(7.7)$ & Amikacin & $15(6.4)$ & Cefotaxime & $10(8.2)$ & Metronidazole & $28(9.7)$ & & & Metronidazole & $2(3.4)$ \\
\hline Cefotaxime & $71(5.6)$ & Ampicillin/BLI & $29(5.4)$ & Metronidazole & $14(6.0)$ & Ampicillin & $9(7.4)$ & Cefazolin & $18(6.2)$ & & & Meropenem & $2(3.4)$ \\
\hline Cefoperazone & $52(4.1)$ & Cefotaxime & $27(5.0)$ & Ceftazidime & $14(6.0)$ & Cefoperazone/ßLI & $6(4.9)$ & Amoxicillin/BLI & $16(5.6)$ & & & Gentamicin & $2(3.4)$ \\
\hline Cefixime & $51(4.0)$ & Gentamicin & $17(3.1)$ & Cefotaxime & $12(5.1)$ & Cefoperazone & $4(3.3)$ & Cefotaxime & $14(4.9)$ & & & Amoxicillin & $2(3.4)$ \\
\hline Ampicillin/ßLI & $44(3.5)$ & Cefixime & $12(2.2)$ & Ciprofloxacin & $9(3.8)$ & Ampicillin/ßLI & $4(3.3)$ & Levofloxacin & $8(2.8)$ & & & & \\
\hline Gentamicin & $42(3.3)$ & Cefoperazone & $11(2.0)$ & Cefoperazone/ßLI & $6(2.6)$ & Amoxicillin & $4(3.3)$ & Gentamicin & $7(2.4)$ & & & & \\
\hline Amikacin & $32(2.5)$ & Amikacin & $10(1.8)$ & Azithromycin & $6(2.6)$ & Levofloxacin & $3(2.5)$ & Cefoperazone/ßLI & $6(2.1)$ & & & & \\
\hline Amoxicillin/ßLI & $31(2.4)$ & Cefoperazone/ßLI & $9(1.7)$ & Tigecycline & $6(2.6)$ & Erythromycin & $3(2.5)$ & Amikacin & $6(2.1)$ & & & & \\
\hline Cefoperazone/ßLI & $28(2.2)$ & Ciprofloxacin & $9(1.7)$ & Cefoperazone & $5(2.1)$ & & & Fosfomycin & $6(2.1)$ & & & & \\
\hline Cotrimoxazole & $28(2.2)$ & Azithromycin & $9(1.7)$ & Ampicillin/ßLI & $5(2.1)$ & & & Ampicillin/ $\beta\llcorner I$ & $5(1.7)$ & & & & \\
\hline Ceftazidime & $21(1.6)$ & Amoxicillin/BLI & $8(1.5)$ & Amoxicillin $\beta \mathrm{LI}$ & $5(2.1)$ & & & Ampicillin & $4(1.4)$ & & & & \\
\hline Ciprofloxacin & $21(1.6)$ & Moxifloxacin & $8(1.5)$ & Fosfomycin & $5(2.1)$ & & & Cefadroxil & $4(1.4)$ & & & & \\
\hline Cefazolin & $18(1.5)$ & & & Piperacillin/ßLI & $5(2.1)$ & & & & & & & & \\
\hline Ampicillin & $17(1.3)$ & & & Doripenem & $4(1.7)$ & & & & & & & & \\
\hline Amoxicillin & $16(1.3)$ & & & & & & & & & & & & \\
\hline Azithromycin & $16(1.3)$ & & & & & & & & & & & & \\
\hline Clindamycin & $16(1.3)$ & & & & & & & & & & & & \\
\hline
\end{tabular}

Figure 3. Systemic antibiotic use by indication based on AWaRe classification. Data are expressed as numbers (percentage). Antibacterial prescriptions for systemic use (J01) were included. DU 90\%, the number of drugs which account for $90 \%$ of the prescriptions; CAI, communityacquired infection; HAI, hospital-acquired infection; $\beta$ LI, $\beta$-lactamase inhibitor.

Table 4. Quality indicators for antibiotic prescribing, by indication

\begin{tabular}{|c|c|c|c|c|c|c|c|c|c|}
\hline \multirow[b]{2}{*}{$\begin{array}{l}\text { Quality } \\
\text { indicators }\end{array}$} & \multirow[b]{2}{*}{$\begin{array}{c}\text { Total } \\
(n=1273)\end{array}$} & \multicolumn{3}{|c|}{ Therapeutic use $(n=777)$} & \multirow[b]{2}{*}{$\begin{array}{c}\text { HAI } \\
\text { targeted } \\
(n=63)\end{array}$} & \multicolumn{2}{|c|}{ Prophylactic use $(n=410)$} & \multirow[b]{2}{*}{$\begin{array}{c}\text { Other } \\
\text { indication }^{a} \\
(n=27)\end{array}$} & \multirow[b]{2}{*}{$\begin{array}{l}\text { Unknown } \\
\text { indication } \\
(n=59)\end{array}$} \\
\hline & & $\begin{array}{l}\text { CAI } \\
\text { empirical } \\
(n=498)\end{array}$ & $\begin{array}{c}\text { CAI } \\
\text { targeted } \\
(n=44)\end{array}$ & $\begin{array}{c}\text { HAI } \\
\text { empirical } \\
(n=172)\end{array}$ & & $\begin{array}{c}\text { Medical } \\
\text { prophylaxis } \\
(n=122)\end{array}$ & $\begin{array}{c}\text { Surgical } \\
\text { prophylaxis } \\
(n=288)\end{array}$ & & \\
\hline Reason documented & $808(63.5)$ & $321(64.5)$ & $35(79.5)$ & $145(84.3)$ & $45(71.4)$ & $58(47.5)$ & $172(59.7)$ & $21(77.8)$ & $11(18.6)$ \\
\hline $\begin{array}{l}\text { Stop/review date } \\
\text { documented }\end{array}$ & $194(15.2)$ & 69 (13.9) & 7 (15.9) & $43(25.0)$ & $18(28.6)$ & $15(12.3)$ & $38(13.2)$ & $2(7.4)$ & $2(3.4)$ \\
\hline $\begin{array}{l}\text { Treatment duration } \\
\text { documented }\end{array}$ & $125(9.8)$ & $35(7.0)$ & $5(11.4)$ & $31(18.0)$ & $12(19.0)$ & $3(2.5)$ & $35(12.2)$ & $0(0.0)$ & $4(6.8)$ \\
\hline \multicolumn{10}{|l|}{ Guideline compliance } \\
\hline yes & $478(37.5)$ & $223(44.8)$ & $17(38.6)$ & 79 (45.9) & $33(52.4)$ & 23 (18.9) & $82(28.5)$ & $21(77.8)$ & $0(0.0)$ \\
\hline no & $378(29.7)$ & $136(27.3)$ & $16(36.4)$ & $70(40.7)$ & $20(31.7)$ & $6(4.9)$ & $124(43.1)$ & $6(22.2)$ & $0(0.0)$ \\
\hline not assessable ${ }^{b}$ & $358(28.1)$ & 139 (27.9) & $11(25.0)$ & $23(13.4)$ & $10(15.9)$ & $93(76.2)$ & $82(28.5)$ & $0(0.0)$ & $0(0.0)$ \\
\hline indication unknown & $59(4.6)$ & $0(0.0)$ & $0(0.0)$ & $0(0.0)$ & $0(0.0)$ & $0(0.0)$ & $0(0.0)$ & $0(0.0)$ & $59(100)$ \\
\hline \multicolumn{10}{|c|}{ Route of administration } \\
\hline parenteral (IV) & $1084(85.2)$ & $444(89.2)$ & $42(95.5)$ & $150(87.2)$ & $58(92.1)$ & $92(75.4)$ & $237(82.3)$ & $10(37.0)$ & $51(86.4)$ \\
\hline oral & $183(14.4)$ & $52(10.4)$ & $2(4.6)$ & $22(12.8)$ & $5(7.9)$ & $30(24.6)$ & $51(17.7)$ & $13(48.2)$ & $8(13.6)$ \\
\hline IV to oral switch & $48(26.2)$ & $6(11.5)$ & $1(50.0)$ & 9 (40.9) & $1(20.0)$ & $1(3.3)$ & $28(54.9)$ & $0(0.0)$ & $2(25.0)$ \\
\hline other & $6(0.47)$ & $2(0.40)$ & $0(0.00)$ & $0(0.00)$ & $0(0.00)$ & $0(0.00)$ & $0(0.00)$ & $4(14.81)$ & $0(0.00)$ \\
\hline Culture sample taken ${ }^{c}$ & $344(44.3)$ & $125(25.1)$ & $44(100)$ & $112(65.1)$ & $63(100)$ & - & - & - & - \\
\hline
\end{tabular}

CAI, community-acquired infection; HAI, hospital-acquired infection.

aOther indication included antibiotics prescribed for neurotoxoplasmosis, pulmonary TB and as a motility agent.

${ }^{b}$ Hospital antibiotic guidelines were not available to assess compliance.

'Only applicable to therapeutic use.

reduction for mortality of $35 \% .^{32}$ The reason for poor guideline compliance (52\%) in our survey is uncertain and probably multifactorial, including local resistance patterns, ineffective guideline dissemination and clinical uncertainty with fear of treatment failure. Our findings should trigger further detailed investigations at hospital and country level.
The WHO AWaRe framework offers an attractive metric for LMICs in the absence of validated quality indicators for antibiotic appropriateness, ${ }^{17,33,34}$ and includes a $>60 \%$ national target of total antibiotic consumption in the Access category by $2023 .^{35}$ However, a recent assessment of antibiotic consumption data from 76 countries in 2000-15 found that the global per-capita 
consumption of Watch antibiotics increased by $90.9 \%$, compared with an increase of $26.2 \%$ in Access antibiotics, with disproportionate increases in Watch antibiotic consumption in LMICs (165\% compared with $27.9 \%$ in high-income countries). ${ }^{18}$ Although Indonesia national-level data have not been included in the AWaRe reports to date, ${ }^{35}$ our survey found hospital consumption of Access antibiotics at $28 \%$ to be below the $60 \%$ target, mostly driven by ceftriaxone and levofloxacin use for CAI and HAI. Although these findings could partially be explained by the national health insurance scheme which determines available antibiotics based on the national formulary, ${ }^{36}$ they also highlight significant challenges for AMS.

Limitations of this study are inherent to the cross-sectional PPS design, providing a mere snapshot of the antibiotic situation in the hospital surveyed. Moreover, given that we used a convenient sample of six hospitals in metropolitan Jakarta, which are potentially better resourced than many other hospitals in Indonesia, data are not necessarily representative for all hospitals in Indonesia, urging caution in extrapolating the observed patterns. Indeed, antibiotic prescribing can be influenced by many factors, e.g. patient case-mix, prevalence of different types of infections, AMR patterns and institutional factors.

In conclusion, we observed high levels of parenteral, empirical use of broad-spectrum antibiotics in Indonesian hospitals, and inadequate performance on key quality indicators of prescribing. Despite important progress in AMS, supported by national policies, ${ }^{10,11}$ the study findings highlighted the need to strengthen AMS to increase use of narrower-spectrum antibiotics through culture-guided, targeted treatment and hospital guideline compliance. Further research is needed to understand the complex drivers of antibiotic prescribing, and to develop context-specific and feasible quality improvement strategies to strengthen existing AMS programmes.

\section{Acknowledgements}

We thank the management, research/medical committees and clinical staff of the participating hospitals for their support, and Reinout van Crevel and Rogier van Doorn for useful feedback on the manuscript.

\section{EXPLAIN study group}

Ralalicia Limato, Erni J. Nelwan, Manzilina Mudia, Helio Guterres, Enty Enty, Ifael Y. Mauleti, Maria Mayasari, Iman Firmansyah, May Hizrani, Raph L. Hamers, Anis Karuniawati, Prof Taralan Tambunan, Prof Amin Soebandrio, Prof Henri Verbrugh, Decy Subekti, Iqbal Elyazar, Mutia Rahardjani, Fitria Wulandari, Prof Reinout van Crevel, Rogier van Doorn, Vu Thi Lan Huong, Nga Do Ti Thuy, Sonia Lewycka, Prof Alex Broom.

\section{Funding}

This work was funded by the Wellcome Trust, UK (106680/Z/14/Z).

\section{Transparency declarations}

None to declare.

\section{Author contributions}

E.J.N. and R.L.H. conceived the idea for the study and are the principal investigators. R.L.H. obtained the funding. R.L., E.J.N. and R.L.H. designed the study protocol. M.M., H.G., E.E., I.Y.M., M.M., I.F. and M.H. collected and verified the data, overseen by R.L. R.L., M. Mudia and J.d.B. curated the database and did the analysis, supervised by R.L.H. R.L. and M. Mudia had full access to all study data. R.L., M. Mudia and R.L.H. drafted the paper. All authors critically revised the manuscript, and all authors gave approval for the final version to be published.

\section{Supplementary data}

Appendix S1 and Tables S1 to S9 are available as Supplementary data at JAC-AMR Online.

\section{References}

1 O'Neill J, Antimicrobial Resistance: Tackling a Crisis for the Health and Wealth of Nations. 2014. https://amr-review.org/sites/default/files/AMR\% 20Review\%20Paper\%20-\%20Tackling\%20a\%20crisis\%20for\% 20the\%20health\%20and\%20wealth\%20of\%20nations_1.pdf.

2 Van Boeckel TP, Gandra S, Ashok A et al. Global antibiotic consumption 2000 to 2010: an analysis of national pharmaceutical sales data. Lancet Infect Dis 2014; 14: 742-50.

3 Goossens H, Ferech M, Vander Stichele R et al. Outpatient antibiotic use in Europe and association with resistance: a cross-national database study. Lancet 2005; 365: 579-87.

4 Klein EY, Van Boeckel TP, Martinez EM et al. Global increase and geographic convergence in antibiotic consumption between 2000 and 2015. Proc Natl Acad SciU S A 2018; 115: E3463-70.

5 WHO. Global Action Plan on Antimicrobial Resistance. 2015. https://apps. who.int/iris/bitstream/handle/10665/193736/9789241509763_eng.pdf? sequence $=1$.

6 Mboi N, Murty Surbakti I, Trihandini I et al. On the road to universal health care in Indonesia, 1990-2016: a systematic analysis for the Global Burden of Disease Study 2016. Lancet 2018; 392: 581-91.

7 Zellweger M, Carrique-mas J, Limmathurotsakul D et al. A current perspective on antimicrobial resistance in Southeast Asia. J Antimicrob Chemother 2017; 72: 2963-72.

8 Coker RJ, Hunter BM, Rudge JW et al. Emerging infectious diseases in southeast Asia: regional challenges to control. Lancet 2011; 377: 599-609.

9 Parathon $\mathrm{H}$, Kuntaman K, Widiastoety TH et al. Progress towards antimicrobial resistance containment and control in Indonesia. BMJ 2017; 358 31-5.

10 Ministry of Health Republic of Indonesia. Minister of Health Republic of Indonesia Policy No. 8 Year 2015. https://pamki.or.id/download/peraturanmenteri-kesehatan-republik-indonesia-nomor-8-tahun-2015-tentangprogram-pengendalian-resistensi-antimikroba-di-rumah-sakit/.

11 Hospital Accreditation Committee. Standard Hospital Accreditation 1st Edition. 2017. http://www.pormiki-dki.org/2016-04-20-03-11-28/daftarbuku-kumpulan-peraturan/84-standar-nasional-akreditasi-rs-snarsed-1-tahun-2017.

12 Ministry of Health Republic of Indonesia. General Guideline of Antibiotic Use. 2011. https://farmalkes.kemkes.go.id/2014/03/pedoman-umum-pen ggunaan-antibiotik/.

13 WHO. WHO Methodology for Point Prevalence Survey on Antibiotic Use in Hospitals Version 1.1. 2018. https://apps.who.int/iris/bitstream/handle/ 10665/280063/WHO-EMP-IAU-2018.01-eng.pdf?ua=1.

14 Versporten A, Bielicki J, Drapier N et al. The worldwide antibiotic resistance and prescribing in European children (ARPEC) point prevalence survey: developing hospital-quality indicators of antibiotic prescribing for children. J Antimicrob Chemother 2016; 71: 1106-17. 
15 Versporten A, Zarb P, Caniaux I et al. Antimicrobial consumption and resistance in adult hospital inpatients in 53 countries: results of an internetbased global point prevalence survey. Lancet Glob Heal 2018; 6: e619-29.

16 Saleem Z, Hassali MA, Godman B et al. Point prevalence surveys of antimicrobial use: a systematic review and the implications. Expert Rev Anti Infect Ther 2020; 18: 897-910.

17 WHO. WHO Releases the 2019 AWaRe Classification Antibiotics. 2019. https://www.who.int/medicines/news/2019/WHO_releases2019AWaRe_clas sification_antibiotics/en/.

18 Klein EY, Milkowska-Shibata M, Tseng KK et al. Assessment of WHO antibiotic consumption and access targets in 76 countries, 2000-15: an analysis of pharmaceutical sales data. Lancet Infect Dis 2021; 21: 107-15.

19 Goossens H. Global Point Prevalence Survey of Antimicrobial Consumption and Resistance (2018 Global-PPS): Protocol. 2018: 26. https:// www.global-pps.com/.

20 Thamlikitkul V, Rattanaumpawan P, Sirijatuphat R et al. Integrated oneday surveillance of antimicrobial use, antimicrobial consumption, antimicrobial resistance, healthcare-associated infection, and antimicrobial resistance burden among hospitalized patients in Thailand. J Infect 2020; 81: 98-106.

21 Singh SK, Sengupta S, Antony R et al. Variations in antibiotic use across India: multi-centre study through Global Point Prevalence survey. J Hosp Infect 2019; 103: 280-3.

22 Hadi U, Kuntaman K, Qibtiyah M et al. Problem of antibiotic use and antimicrobial resistance in Indonesia: are we really making progress? Indones J Trop Infect Dis 2013; 4: 5-8.

23 Saleem Z, Hassali MA, Versporten A et al. A multicenter point prevalence survey of antibiotic use in Punjab, Pakistan: findings and implications. Expert Rev Anti Infect Ther 2019; 17: 285-93.

24 Xie DS, Xiang LL, Li R et al. A multicenter point-prevalence survey of antibiotic use in 13 Chinese hospitals. J Infect Public Health 2015; 8: 55-61.

25 Thu TA, Rahman M, Coffin S et al. Antibiotic use in Vietnamese hospitals: a multicenter point-prevalence study. Am J Infect Control 2012; 40: 840-4.

26 ECDC. Point Prevalence Survey of Healthcare-Associated Infections and Antimicrobial Use in European Acute Care Hospitals. 2013. https://www.ecdc. europa.eu/sites/default/files/media/en/publications/Publications/healthcareassociated-infections-antimicrobial-use-PPS.pdf.
27 Dahesihdewi A, Sugiani AK, Parwati I, Data Surveilans Mikroba Dan Kepekaannya Terhadap Antibiotik Berdasarkan Tipe Rumah Sakit Di Indonesia Tahun 2017. 2018. http://forlabinfeksi.or.id/wp-content/uploads/ 2018/08/Final-Buku-DATA-SURVEILANS-MIKROBA-2018-PatKLIn-201808141.pdf.

28 WHO. Global Guidelines on the Prevention of Surgical Site Infection. 2018. https://apps.who.int/iris/bitstream/handle/10665/250680/9789241549882eng.pdf?sequence $=8$.

29 van den Bosch CMA, Hulscher MEJL, Natsch S et al. Applicability of generic quality indicators for appropriate antibiotic use in daily hospital practice: a cross-sectional point-prevalence multicenter study. Clin Microbiol Infect 2016; 22: 888.e1-9.

30 Malcolm W, Nathwani D, Davey P et al. From intermittent antibiotic point prevalence surveys to quality improvement: experience in Scottish hospitals. Antimicrob Resist Infect Control 2013; 2: 3.

31 Zarb P, Amadeo B, Muller A et al. Identification of targets for quality improvement in antimicrobial prescribing: the web-based ESAC point prevalence survey 2009. J Antimicrob Chemother 2011; 66: 443-9.

32 Schuts EC, Hulscher MEJL, Mouton JW et al. Current evidence on hospital antimicrobial stewardship objectives: a systematic review and meta-analysis. Lancet Infect Dis 2016; 16: 847-56.

33 WHO. Adopt AWaRe: Handle Antibiotics with Care. 2019. https://adopta ware.org/.

34 Sharland M, Pulcini C, Harbarth S et al. Classifying antibiotics in the WHO Essential Medicines List for optimal use—be AWaRe. Lancet Infect Dis 2018; 18: 18-20.

35 WHO. WHO Report on Surveillance of Antibiotic Consumption: 20162018 Early Implementation. 2018. https://apps.who.int/iris/bitstream/han dle/10665/277359/9789241514880-eng.pdf.

36 Yuniarti E, Prabandari YS, Kristin E et al. Rationing for medicines by health care providers in Indonesia National Health Insurance System at hospital setting: a qualitative study. J Pharm Policy Pract 2019; 12 : 7.

37 Reilly JS, Coignard B, Price L et al. The reliability of the McCabe score as a marker of co-morbidity in healthcare-associated infection point prevalence studies. J Infect Prev 2016; 17: 127-9. 